\title{
Export nucléaire des protéines et homéostasie cellulaire
}

> L'apparition du noyau dans les cellules eucaryotes a imposé la mise en place de mécanismes

Catherine Dargemont spécifiques permettant d'utiliser et de protéger l'information génétique mais également de coordonner les fonctions nucléaires et cytoplasmiques. Les études menées au cours des 10 dernières années ont permis d'élaborer un modèle selon lequel les molécules importées vers le noyau ou exportées vers le cytoplasme présentent des séquences d'adressage reconnues par des récepteurs spécifiques de transport nucléaire. Ces interactions sont orchestrées par la petite GTPase Ran qui assure la directionnalité des échanges nucléocytoplasmiques. Cet article tentera de faire le point des connaissances sur les mécanismes d'export nucléaire des protéines et le rôle de ces voies de transport dans la régulation d'autres fonctions cellulaires. <

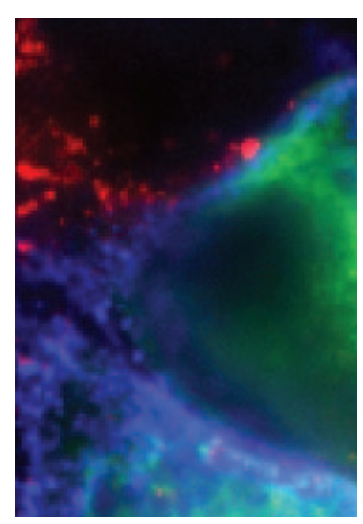

diffuser librement à travers le pore nucléaire. En revanche la translocation de molé-

cules de poids moléculaire supérieur à $40 \mathrm{kDa}$, c'est-àdire la plupart des protéines et des complexes ribonucléo-protéiques, mais également de certaines protéines de poids moléculaire inférieur à $40 \mathrm{kDa}$ comme les histones, s'opère de façon active à travers le canal central dont le diamètre peut atteindre $25 \mathrm{~nm}$.

Les substrats d'import ou d'export nucléaire sont identifiés grâce à des séquences d'adressage spécifiques par des récepteurs appartenant pour la plupart à une même famille de protéines, les karyophérines $\beta$. La capacité de ces récepteurs d'interagir avec certaines nucléoporines leur permet de cibler leurs substrats au pore nucléaire et d'assurer leur translocation. Une fois dans le compartiment d'arrivée, les substrats transportés sont libérés et les récepteurs sont recyclés afin de permettre un nouveau cycle de transport. L'interaction entre substrat et récepteur est contrôlée par la petite GTPase Ran appartenant à la superfamille Ras (Figure 1) $(\rightarrow)$. La répartition nucléocytoplasmique des protéines qui contrôlent le cycle du GTP associé à Ran est telle qu'il existe dans la cellule un gradient de Ran-GDP (cytoplasmique) /Ran- 
GTP (nucléaire) de part et d'autre de l'enveloppe nucléaire qui conditionne l'orientation des échanges nucléocytoplasmiques: les récepteurs d'import nucléaire (importines ou karyophérines) se lient à leurs substrats en l'absence de Ran-GTP (dans le cytoplasme) et les libèrent en présence de Ran-GTP (dans le noyau) alors que les récepteurs d'export (exportines) ne reconnaissent leurs substrats qu'en présence de Ran-GTP (dans le noyau) et les libèrent lors de l'hydrolyse du GTP (dans le cytoplasme) [1].

\section{Rétention nucléaire}

Dès 1958, les travaux de Goldstein fondés sur la transplantation nucléaire chez l'amibe permettaient de mettre en évidence l'existence de protéines qui font la navette entre le noyau et le cytoplasme [2]. Chez les eucaryotes supérieurs, l'identification des protéines-navettes ainsi que la caractérisation de leur voie d'export nucléaire a dû attendre l'établissement de deux approches expérimentales fondées sur la formation d'hétérokaryons inter-espèces et la micro-injection.

La capacité d'une protéine nucléaire de sortir du noyau dépend tout d'abord de la force de ses interactions avec d'autres composants nucléaires. Ainsi, la protéine hnRNP C ou la lamine $B$, qui sont très fortement ancrées dans le noyau, ne sont jamais exportées. En revanche, toute protéine non retenue peut être transportée transitoirement vers le cytoplasme. Dans la plupart des cas, il s'agit d'un export nucléaire lent, peu efficace, dont le rôle, à l'heure actuelle, n'est pas établi et qui dépend de l'affinité de ces protéines pour les composants nucléaires $[3,4]$.

\section{Séquences d'export nucléaire}

Certaines protéines cellulaires ou virales telles hnRNPAl ou la protéine Rev du virus VIH-l présentent, en revanche, la capacité de sortir très rapidement du noyau. Des études de cartographie moléculaire menées en parallèle sur différentes protéines transportées rapidement du noyau vers le cytoplasme ont permis l'identification de séquences spécifiques d'export nucléaire (NES). Ainsi la protéine hnRNPAl, qui s'associe aux ARN pré-messagers dans le noyau et accompagne les ARNm lors de leur export, possède un motif de 39 acides aminés (dénommé M9) qui lui confère la double capacité d'import et d'export nucléaire [5]. La séquence d'export nucléaire la mieux caractérisée à l'heure actuelle et présente dans un nombre important de protéines est constituée d'un motif hydrophobe d'une dizaine d'acides aminés riches en leucines, résidus critiques dans la fonction de transport $[6,7]$. Cette séquence est nécessaire et suffisante pour l'export nucléaire. Fusionnée à une protéine rapporteur, elle lui confère une capacité d'export nucléaire rapide. L'existence de récepteurs spécifiques de ces séquences NES était suggérée par l'observation que cette voie de transport pouvait être saturée. L'identification de ces récepteurs a été facilitée par l'observation qu'une drogue, la leptomycine $B$, était capable d'inhiber l'export des protéines portant ces motifs de transport [8].

\section{Récepteurs d'export nucléaire}

Des modèles expérimentaux distincts ont permis de montrer que les séquences NES riches en leucine sont reconnues par un récepteur spécifique, cible de la leptomycine $B$ et apparenté à la famille des karyophérines $\beta, \mathrm{CRMI}$ ou exportine 1 [9-12]. D'autres récepteurs d'export nucléaire des protéines ont alors été caractérisés et il est rapidement apparu que les récepteurs d'export (exportines) et d'import (importines) nucléaire appartiennent à une même famille de protéines (Tableau I). En particulier,

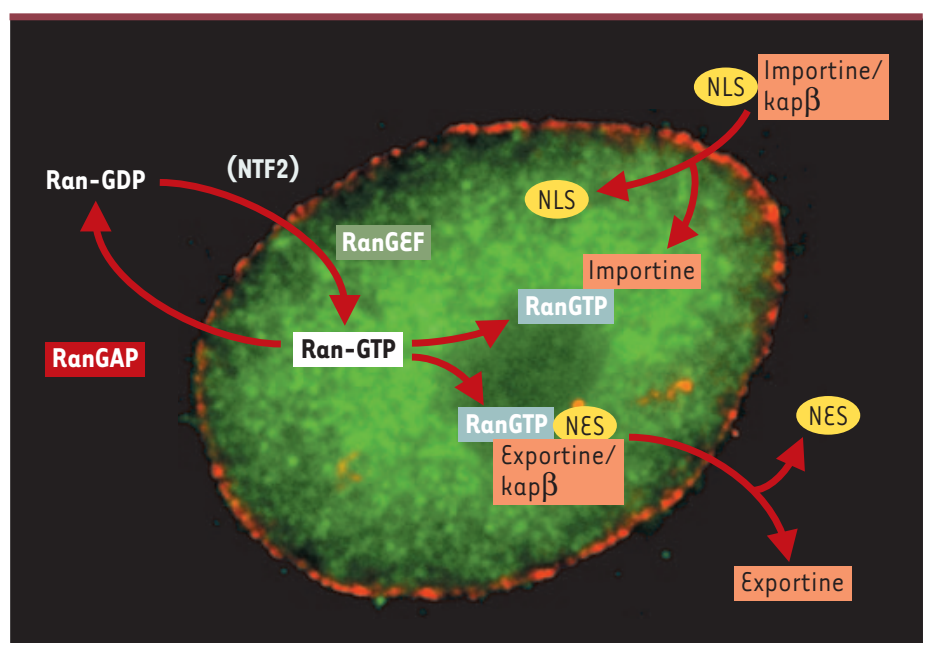

Figure 1. Cycle de Ran et transport nucléaire. Les substrats d'import ou d'export nucléaire sont reconnus grâce à des séquences d'adressage spécifiques (NLS ou NES) par des récepteurs appartenant à une même famille de protéines, les karyophérines $\beta$ (Kap $\beta$ ). Ces protéines interagissent avec le substrat, le pore nucléaire et la GTPase Ran. Le facteur d'échange de Ran, ou RanGEF (en vert) est exclusivement nucléaire alors que Ran-GAP (en rouge) est exclusivement cytoplasmique et est concentré au niveau du pore nucléaire. Cette répartition engendre, de part et d'autre de l'enveloppe nucléaire, un gradient de Ran-GDP (cytoplasmique)/Ran-GTP (nucléaire) responsable de l'orientation des échanges nucléocytoplasmiques: les récepteurs d'import nucléaire (importines) se lient à leurs substrats en l'absence de Ran-GTP (dans le cytoplasme) et les libèrent en présence de Ran-GTP (dans le noyau) alors que les récepteurs d'export (exportines) se lient à leurs substrats en présence de Ran-GTP (dans le noyau) et les libèrent lors de l'hydrolyse du GTP (dans le cytoplasme). NLS : nuclear localisation signal ; NES : nuclear export signal. 
la protéine CAS (Cselp chez la levure) est responsable de l'export nucléaire des karyophérines $\alpha$ (protéines se liant aux signaux d'import nucléaire) et permet ainsi au récepteur qui a déchargé son substrat dans le noyau d'être recyclé dans le cytoplasme [13]. De même, l'exportine 4 permet l'export du facteur d'initiation de la traduction elF-5A, qui semble être son seul substrat [14]. L'exportine 5 , décrite très récemment, semble être plus particulièrement impliquée dans l'export nucléaire de protéines interagissant avec les ARN double-brin [15]. Enfin, les récepteurs Msn5 (probable homologue de l'exportine 5 chez la levure) et importine 13 ont pour particularité de fonctionner comme récepteurs d'import pour certains substrats et récepteurs d'export pour d'autres $[16,17]$. Récemment, des données inattendues ont montré que la calréticuline, une protéine de liaison au calcium, est responsable du transport des récepteurs nucléaires hormonaux du noyau vers le cytoplasme [18]. La calréticuline reconnaît un motif localisé dans le domaine de liaison à l'ADN et, bien que cette protéine ne soit pas une karyophérine $\beta$, son interaction avec le récepteur des glucocorticoïdes est contrôlée par RanGTP. Il est donc actuellement concevable que des signaux d'export nucléaire puissent être reconnus par des récepteurs de type différent mais qui utilisent le même mode de fonctionnement.

\section{Mécanismes de translocation à travers le pore nucléaire}

L'assemblage des complexes exportine/substrat est orchestré par la GTPase Ran. Comme toutes les GTPases,

\begin{tabular}{|c|c|c|}
\hline Vertébrés & Levure & Substrat \\
\hline \multicolumn{3}{|l|}{ Exportines } \\
\hline $\begin{array}{l}\text { CRM1 } \\
\text { (exportine 1) }\end{array}$ & CRMI (Xpolp) & $\begin{array}{l}\text { Protéines contenant } \\
\text { un NES riche en leucines }\end{array}$ \\
\hline CAS & CSEl (Kap109p) & Importine $\alpha$ \\
\hline \multirow[t]{2}{*}{ Exportine 4} & & elF-5A \\
\hline & Msn5 (Kap142p) & $\begin{array}{l}\text { Substrats d'export: Pho4, Ste5, Farl } \\
\text { Substrats d'import: RPA }\end{array}$ \\
\hline Exportine 5 & Msn5? & $\begin{array}{l}\text { Protéines se liant aux ARN } \\
\text { double brins (ILF3, PKR...) }\end{array}$ \\
\hline Importine 13 & & $\begin{array}{l}\text { Substrats d'export: elFlA } \\
\text { Substrats d'import: Ubc9, yl4 }\end{array}$ \\
\hline \multicolumn{3}{|l|}{ Autres } \\
\hline Calréticuline & & $\begin{array}{l}\text { Récepteurs nucléaires } \\
\text { (glucocorticoïdes, vitamine D...) }\end{array}$ \\
\hline
\end{tabular}

Table I. Récepteurs d'export nucléaire de levure et de vertébrés et leurs substrats.
Ran possède une activité GTPase intrinsèque faible stimulée par la RanGAP 1 (GTPase activating protein 1) et les co-facteurs RanBPl et RanBP2 (Ran binding proteins), alors que l'échange du GDP en GTP est stimulé par un facteur d'échange de nucléotide ou RanG\&F. Ces facteurs régulateurs du cycle de Ran présentent une compartimentation intracellulaire très bien définie (Figure 1). RanGAP1, RanBPl et RanBP2 sont essentiellement cytoplasmiques ou associés à la face cytoplasmique du pore nucléaire et maintiennent donc Ran sous forme liée au GDP dans le cytoplasme. Le facteur NTF2 (nuclear transport factor 2) se lie directement à RanGDP et facilite son accumulation dans le noyau, compartiment dans lequel RanG\&F, protéine nucléaire associée à la chromatine, échange le GDP en GTP. Ainsi se crée un gradient de RanGDP (cytoplasmique) /RanGTP (nucléaire) de part et d'autre de l'enveloppe nucléaire [19].

Les exportines interagissent avec RanGTP dans le noyau mais la formation de ce complexe dépend de la présence du substrat à exporter. Inversement, la reconnaissance d'un substrat d'export par une exportine requiert la présence de RanGTP. Les modèles de reconstitution de l'export nucléaire in vitro permettent d'affirmer que les protéines CRMl et RanGTP sont nécessaires et suffisantes pour assembler un complexe d'export nucléaire. Cependant, des données récentes indiquent que certaines protéines faciliteraient cette étape d'assemblage (Figure 2). En particulier, la protéine RanBP3 peut former un complexe trimérique avec les protéines CRMI et RanGTP indépendamment de la séquence NES et permettrait de former un pré-complexe d'export incapable d'interagir avec le pore nucléaire tant qu'un substrat contenant un NES n'a pas été reconnu [20].

Bien qu'il n'ait pas été possible expérimentalement de découpler les réactions de translocation et d'ancrage des complexes d'export au pore nucléaire, il est clair que ces étapes de l'export nucléaire impliquent des interactions directes et spécifiques entre exportines et certaines protéines du pore nucléaire. Ces protéines, impliquées dans l'export mais aussi dans l'import nucléaire, sont appelées nucléoporines FG parce qu'elles possèdent des domaines comportant de nombreuses répétitions d'acides aminés phénylalanine (F) et glycine (G) reconnues par les karyophérines $\beta$. La distribution homogène de ces protéines au niveau du transporteur central mais également à la périphérie du pore nucléaire suggère que des cycles consécutifs d'association/dissociation entre les nucléoporines FG et les complexes de transport pourraient permettre leur translocation [21]. Cette hypothèse est renforcée par l'identification récente des nucléoporines de levure et d'un grand nombre de nucléoporines de mammifères, 
leur localisation au sein du pore nucléaire et leur capacité d'interaction avec les différentes exportines [22]. De plus, la distribution asymétrique de certaines nucléoporines de part et d'autre du pore nucléaire suggère un rôle de ces protéines dans l'orientation du transport nucléocytoplasmique [23]. Récemment, des études cinétiques précises ont conduit à l'élaboration d'un modèle séduisant selon lequel la concentration élevée de motifs FG au niveau du canal central et la capacité d'attraction mutuelle (interactions hydrophobes) des groupements phénylalanine créeraient une barrière sélective que seules les molécules capables d'interagir avec les motifs FG, et donc de détruire localement ces attractions mutuelles, pourraient traverser [24]. En faveur de ce modèle, il faut noter que lorsque le gradient de RanGTP de part et d'autre de l'enveloppe nucléaire est maintenu, la translocation à travers le pore peut avoir lieu en l'absence d'hydrolyse d'ATP.

Parvenus sur la face cytoplasmique du pore nucléaire, les complexes d'export doivent être dissociés afin de permettre la libération du substrat dans le cytoplasme et le recyclage du récepteur. Ainsi qu'il a été mentionné précédemment, cette étape est contrôlée par les protéines RanBPl et RanBP2 qui favorisent la dissociation de RanGTP, probablement par déstabilisation de l'interaction entre exportine et substrat. Les protéines RanBPl et RanBP2 présentent en effet des domaines similaires de liaison à RanGTP, dissocient RanGTP de CRMl in vitro et permettent à RanGAP de stimuler l'hydrolyse de RanGTP en RanGDP, rendant ainsi la réaction de dissociation irréversible puisque les exportines n'ont aucune affinité pour RanGDP [25]. Des données récentes indiquent que la protéine NXT1 (NTF2-related export protein 1) participerait à cette étape de dissociation. Cette protéine, localisée au pore nucléaire, est capable d'interagir avec de nombreuses nucléoporines in vitro et stimule différentes voies d'export nucléaire. Elle interagirait avec les complexes d'export avant leur arrivée sur la face cytoplasmique du pore et, soit optimiserait leur structure en vue de la dissociation, soit les dirigerait vers un site précis partiment nucléaire. du pore nucléaire au niveau duquel la dissociation peut avoir lieu [26]. Notons que RanBP2 constitue un excellent candidat du site de dissociation puisque: (1) elle est elle-même une nucléoporine localisée sur les fibrilles cytoplasmiques du pore; (2) elle présente des sites de reconnaissance de Ran et des exportines; et, enfin, (3) elle permet de concentrer RanGAP à la sortie du pore en interagissant spécifiquement avec la forme sumoylée majoritaire de RanGAP [27-29]. Secondairement à la dissociation du complexe d'export, les exportines et NXTl sont recyclées vers le noyau, RanGDP est reconnu par NTF2 et réimporté, et les substrats sont libérés dans le cytoplasme (Figure 2).

\section{Régulation de l'export nucléaire et rôles dans la coordination des fonctions nucléaires et cytoplasmiques}

Bien que la régulation des mécanismes d'export nucléaire puisse en principe s'exercer à chaque étape et sur chaque protagoniste du transport, la plupart des études actuelles montrent que la modulation de l'interaction entre substrat et récepteur s'exerce principalement via l'accessibilité des séquences de transport nucléaire. L'accessibilité de ces séquences à leur récepteur est déterminée: (1) par leur localisation au sein de la structure tridimensionnelle du substrat ; (2) par des modifications post-traductionnelles; et (3) par l'inter-

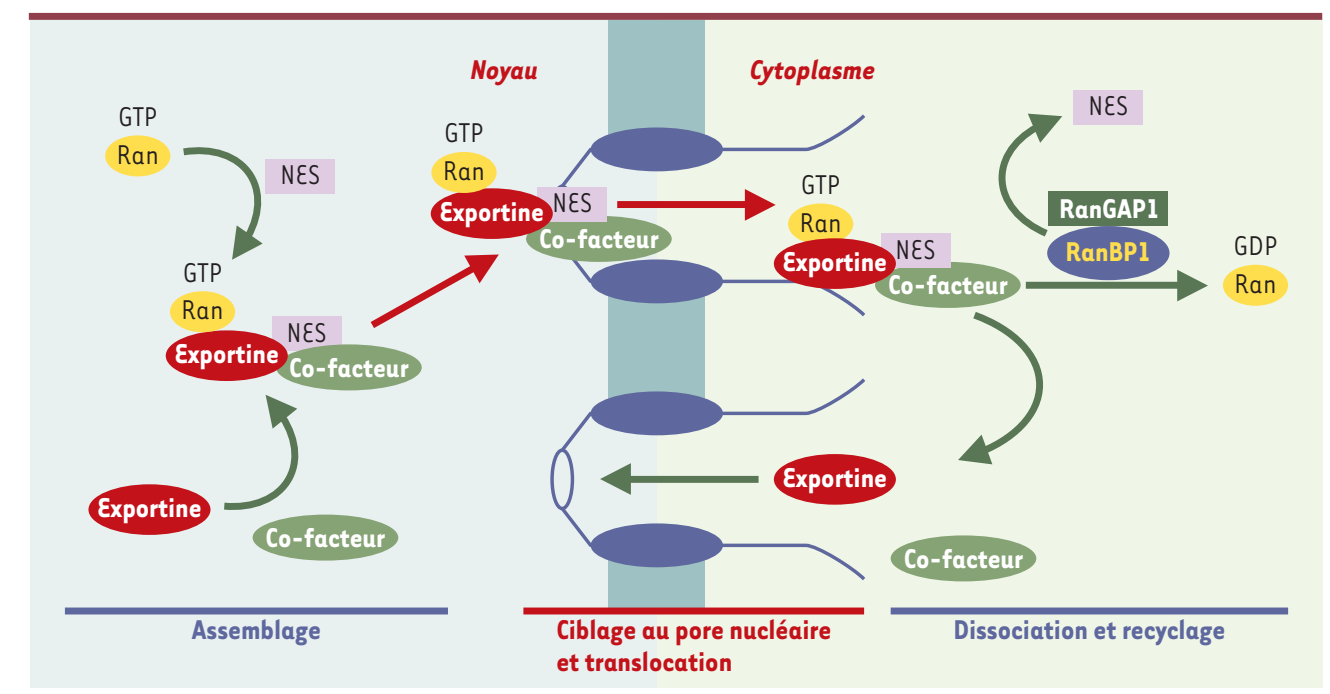

Figure 2. Schéma récapitulatif des différentes étapes de l'export nucléaire des protéines. Les protéines nucléaires contenant un NES sont reconnues par un récepteur, ou exportine, en présence de RanGTP. Ce complexe, dont la formation est éventuellement facilitée par un co-facteur, est alors ciblé au pore nucléaire qu'il traverse grâce à l'interaction entre l'exportine et les nucléoporines. Dans le cytoplasme, l'action concertée des protéines RanBPI (et RanBP2) et Ran GAP stimule l'hydrolyse du GTP sur Ran et permet ainsi la dissociation du complexe d'export. La protéine exportée est alors libérée dans le cytoplasme alors qu'exportine et co-facteur sont recyclés dans le com- 
action avec des partenaires protéiques qui peuvent masquer ou démasquer ces séquences. La régulation de l'accessibilité des motifs de transport permet de moduler la répartition nucléocytoplasmique d'un grand nombre de facteurs cellulaires impliqués notamment dans les voies de signalisation, la progression du cycle cellulaire, le métabolisme des ARN, la polarité cellulaire, et apparaît clairement comme une voie majeure du contrôle de l'homéostasie des cellules eucaryotes.

Ainsi, la localisation des facteurs de transcription dans le compartiment nucléaire, étape indispensable à leur activité, est dans bien des cas soumis à une régulation très stricte. Par exemple, l'activité transcriptionnelle du facteur de transcription NF- $K B$, impliqué dans les réponses immunitaires, inflammatoires et anti-apoptotiques, est essentiellement contrôlée par son interaction avec les protéines $I K B$, capables de contrôler strictement la localisation sub-cellulaire de ce facteur de transcription [30]. Dans la plupart des cellules non stimulées, l'interaction entre $N F-\kappa B$ et $\mid \kappa B \alpha$ masque la séquence de localisation nucléaire de $N F-\kappa B$, et empêche donc son transport dans le noyau (Figure 3). La stimulation des cellules par divers agents, comme le TNF

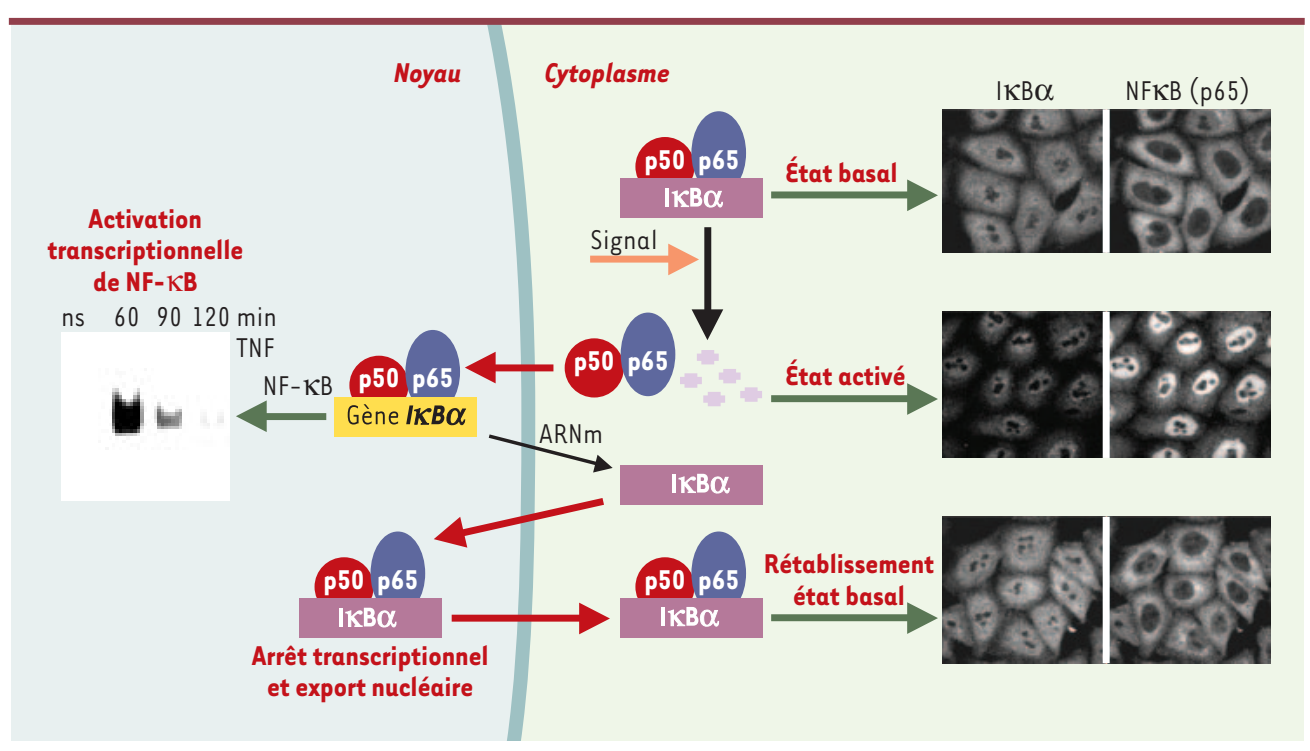

Figure 3. Transport nucléaire et régulation du facteur de transcription NF- KB. Dans les cellules non stimulées, à l'état basal, le facteur de transcription NF- KB (p50 et p65) est retenu sous une forme inactive dans le cytoplasme par son interaction avec $\mid \kappa B \alpha$, qui masque le signal de localisation nucléaire de $N F-\kappa B$. Cela est visualisé par détection par immunofluorescence de NF-KB dans le cytoplasme des cellules. Lorsque les cellules sont activées, IKB est phosphorylé puis dégradé. NF-KB est alors transporté dans le noyau où il active la transcription de gènes spécifiques, dont le gène d'IKB $\alpha$. La disparition cytoplasmique de $\mid \kappa B \alpha$ et la translocation nucléaire de NF- $K B$ sont clairement détectées par immunofluorescence. $I \kappa B \alpha$ est capable, notamment lors de cette néosynthèse, d'être importé dans le noyau et inhibe alors l'interaction de NF-KB avec l'ADN avant de promouvoir un export rapide de NF-KB vers le cytoplasme. Cette boucle de régulation participe à la restauration de l'état basal lorsque les cellules ne reçoivent plus de signal d'activation et contribue, en toute circonstance, à adapter le taux d'activité transcriptionnelle de NF-KB aux besoins de la cellule. ou l'interleukine-1, engendre l'activation en cascade d'une série de kinases et conduit à la phosphorylation $d^{\prime} \mid \kappa B \alpha$, qui est alors ubiquitinylé puis dégradé par le protéasome. Le NF-KB libéré est transporté dans le noyau où il active la transcription de nombreux gènes, dont le gène $d^{\prime} \mid \kappa B \alpha$, induisant ainsi une resynthèse rapide de son inhibiteur. Cette néosynthèse est corrélée à l'apparition massive d'| $\kappa B \alpha$ dans le noyau, compartiment dans lequel $I \kappa B \alpha$ interagit avec $N F-\kappa B$, inhibe sa liaison aux séquences d'ADN spécifiques et provoque l'arrêt de la transcription de gènes contrôlés par NF-KB. $\mid \kappa B \alpha$ induit alors un export rapide du complexe NF$\kappa B / I \kappa B \alpha$ du noyau vers le cytoplasme grâce à deux séquences d'export nucléaire reconnues par le transporteur CRMl et contribue ainsi au rétablissement de l'état basal dans les cellules [31-33]. Mais l'export nucléaire d' $\mid \kappa B \alpha$ participe également à l'activation du facteur NF- $\kappa B$. En effet, dans le noyau, $I \kappa B \alpha$ ne peut être ni phosphorylé ni dégradé en réponse à un signal d'activation et doit donc être exporté vers le cytoplasme pour y être dégradé et permettre une activation optimale du facteur de transcription [34]. Contrairement à l'idée originelle selon laquelle la localisation de NF- $\kappa B$ dans le noyau était strictement dépendante de l'activation cellulaire, il semble maintenant plus vraisemblable que les protéines $N F-\kappa B$ et $\mid \kappa B \alpha$ fassent continuellement la navette entre noyau et cytoplasme, et que leurs niveaux relatifs dans chaque compartiment résultent non seulement du niveau d'expression $d^{\prime} \mid \kappa B \alpha$ mais aussi d'un équilibre entre import et export nucléaire.

Chez les eucaryotes, la progression du cycle cellulaire est gouvernée par des boucles régulatrices d'activation/inactivation des complexes cycline/ Cdk (cyclin dependent kinase) dont l'expression temporelle est extrêmement précise. Alors qu'il est clairement établi que l'activité des complexes cycline/Cdk est contrôlée par la dégradation, l'état de phosphorylation et la présence de peptides 
inhibiteurs, il est apparu au cours des dernières années que la régulation de leur localisation sub-cellulaire est essentielle pour assurer une coordination correcte du cycle cellulaire [35]. Ainsi, l'accumulation du complexe Cdc2-cycline Bl dans le noyau pendant la prophase est indispensable à la mise en place et à la coordination des événements mitotiques. Bien que ce complexe soit synthétisé et assemblé pendant l'interphase, la présence d'une séquence NES reconnue par CRMI dans la cycline Bl permet d'exclure Cdc2-cyclineBl du noyau et de maintenir ce complexe dans un état inactif dans le cytoplasme en interphase [36-38]. Lors de la transition G2/M, la phosphorylation d'un résidu sérine du NES de la cycline Bl provoque une inhibition de l'export nucléaire et une accumulation du complexe dans le noyau [39]. Par ailleurs, l'entrée en mitose requiert également l'activation de Cdc2 par la phosphatase Cdc25. Là encore, les effets concertés de l'export nucléaire par la voie CRMl et de l'interaction avec les protéines 14-3-3, qui restreignent l'import nucléaire et empêchent $\mathrm{Cdc} 25$ de s'associer à $\mathrm{Cdc} 2$, induisent une rétention cytoplasmique efficace de Cdc25 inactive en interphase $[40,41]$. La déphosphorylation de $\mathrm{Cdc} 25$ pendant la prophase entraîne une accumulation nucléaire de la phosphatase activée. La régulation du transport nucléaire permet donc de synchroniser activation et localisation sub-cellulaire du complexe Cdc2-cycline Bl (Figure 4). Plus généralement, les échanges nucléocytoplasmiques constants et contrôlés des acteurs du cycle cellulaire permettent d'assurer non seulement une coordination parfaite des événements nucléaires et cytoplasmiques au cours du cycle mais également une surveillance continue des dysfonctionnements ou des dommages qui doivent conduire à un arrêt du cycle.

De même que la rétention dans le cytoplasme de protéines qui exercent leur fonction dans le noyau permet de contrôler leur activité, la rétention nucléaire de facteurs actifs dans le cytoplasme restreint leur action. Ce mode de régulation est utilisé dans le contrôle de la polarité cellulaire chez la levure $S$. cerevisiae. L'orientation du cytosquelette d'actine soit vers le site de bourgeonnement, soit vers un partenaire sécrétant des phéromones lors de la conjugaison afin de s'orienter correctement par rapport au gradient morphogénétique $[42,43]$, est sous le contrôle de l'activation de la GTPase Cdc42p par son facteur d'échange Cdc24p. Or, pendant la phase Gl du cycle cellulaire, Cdc24p est séquestré dans le noyau par la protéine Farl [44]. Lorsque les cellules atteignent une taille critique, le complexe cycline/Cdk Cdc28p-CInp est activé et conduit à la phosphorylation de Farlp qui est alors ubiquitinylée puis dégradée par le protéasome. Cdc24p alors libéré est exporté vers le cytoplasme où il est recruté spécifiquement vers le site de bourgeonnement par la GTPase Budlp $[44,45]$. En revanche, en présence d'un gradient de phéromones, le complexe Cdc28p-CInp est inhibé et Farlp stabilisé [46]. Le complexe FarlpCdc24p est alors transporté vers le cytoplasme grâce au NES de Farlp reconnu par l'exportine Msn5, et Cdc24 est ciblé par Farlp non pas vers le site de bourgeonnement mais vers les récepteurs des phéromones activés [44, 47]. Les différents partenaires d'export nucléaire de Cdc24p contrôlent donc sa localisation subcellulaire et permettent ainsi la polymérisation localisée et polarisée du cytosquelette d'actine.

Bien que la connaissance des mécanismes moléculaires de l'export nucléaire ait connu des progrès fulgurants au cours des dernières années, les mécanismes précis de translocation à travers le pore nucléaire, le déroulement spatio-temporel des différentes étapes et les mécanismes de régulation des machineries d'export restent encore à élucider. Néanmoins, il est aujourd'hui évident que les échanges nucléocytoplasmiques jouent un rôle majeur dans le contrôle de la physiologie cellulaire et surtout qu'une analyse dynamique de la localisation intracellulaire des protéines est indispensable à la compréhension des régulations fines qui permettent à la cellule eucaryote de coordonner les fonctions assurées dans chacun de ses compartiments. $\diamond$

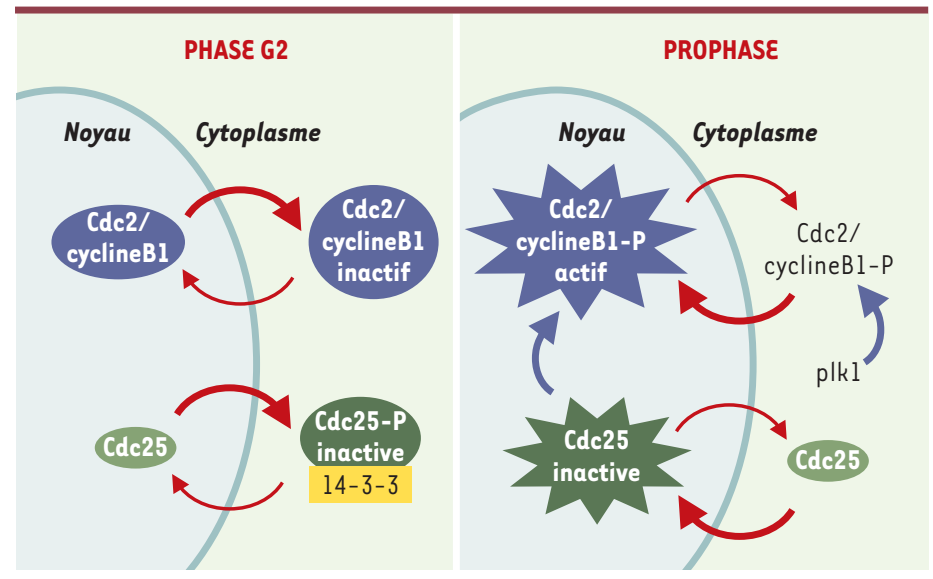

Figure 4. Répartition nucléocytoplasmique du complexe Cdc2-cycline B1 et de la phosphatase Cdc25 pendant la phase $\mathrm{G} 2$ et au cours de la prophase. Durant la phase G2 du cycle cellulaire, un export nucléaire efficace permet de maintenir le complexe Cdc2-cycline Bl inactif dans le cytoplasme. De plus, la phosphatase Cdc25, principal activateur de Cdc2, est séquestrée sous une forme phosphorylée inactive dans le cytoplasme par l'action concertée de son interaction avec les protéines 14-3-3 et d'un export nucléaire rapide. Lors de la prophase, Cdc25 déphosphorylée et activée s'accumule dans le compartiment nucléaire. Dans le même temps, la phosphorylation du NES de la cycline Bl par la polo-like kinase l (plkl) permet une translocation efficace du complexe $\mathrm{Cdc} 2 /$ cycline Bl dans le noyau et inhibe l'export nucléaire. Dans le noyau, Cdc2 est alors activé par Cdc25. 


\section{SUMMARY}

Nuclear export of proteins:

\section{molecular mechanisms and functions}

The nucleus of eukaryotic cells spatially separates DNA replication and RNA transcription from cytoplasmic protein synthesis. Thus, the nuclear membrane must ensure a strict and selective molecular control of exchanges between the nucleus and the rest of the cell, not only to protect and correctly transmit genetic information but also to synchronize nuclear and cytoplasmic functions. Studies over the past ten years led to the identification of targeting sequences (nuclear import or export sequences) recognized by specific receptors, called importins and exportins, which induce transport through the nuclear pore complex. Cargo-receptor interactions are orchestrated by Ran, a small and abundant GTPase. The compartmentalization of the factors that control the GDP- and GTP-bound state of Ran is believed to create a steep gradient of RanGDP (cytoplasmic) / RanGTP (nuclear) concentrations across the nuclear membrane. This gradient controls the directionality of nucleocytoplasmic transport pathways since cargoimportin complexes are induced to disassemble upon binding to RanGTP in the nucleus whereas RanGTP is used to assemble cargo-exportin complexes. In this review, we focus on what is known about the various steps involved in nuclear export of proteins, an intracellular route discovered more than 40 years ago, which has remained largely uncharacterized until recently. Furthermore, the regulation of nuclear transport is now considered one of the most efficient mechanisms to adapt cellular responses to environment conditions by restricting access to nuclear or cytoplasmic compartments. In particular, we illustrate here how nuclear export regulation participates to the control of transcriptional responses, cell cycle progression or the establishment of cell polarity. $\Delta$

\section{RÉFÉRENCES}

1. Mattaj IW, EngImeier L. Nucleocytoplasmic transport: the soluble phase. Annu Rev Biochem 1998; 67: 265-306.

2. Goldstein L. Localization of nucleus-specific protein as shown by transplantation experiments in Amoebæ proteus. Exp Cell Res 1958; 15: 635-7.

3. Schmidt-Zachmann MS, Dargemont C, Kuhn LC, Nigg EA. Nuclear export of proteins: the role of nuclear retention. Cell 1993; 74: 493-504.
4. Nakielny S, Dreyfuss G. The hnRNP C proteins contain a nuclear retention sequence that can override nuclear export signals. J Cell Biol 1996; 134: 1365-73.

5. Michael WM, Choi M, Dreyfuss G. A nuclear export signal in hnRNP Al: a signalmediated, temperaturedependent nuclear protein export pathway. Cell 1995; 83: 415-22

6. Wen W, Meinkoth JL, Tsien Ry, Taylor SS. Identification of a signal for rapid export of proteins from the nucleus. Cell 1995; 82: 463-73.
7. Fischer U, Huber J, Boelens WC, Mattaj IW, Luhrmann R. The HIV-1 Rev activation domain is a nuclear export signal that accesses an export pathway used by specific cellular RNAs. Cell $1995 ; 82: 475-83$.

8. Wolff B, Sanglier JJ, Wang Y. Leptomycin B is an inhibitor of nuclear export: inhibition of nucleocytoplasmic translocation of the human immunodeficiency virus type 1 (HIV-1) Rev protein and Rev-dependent mRNA. Chem Biol 1997; 4 : 139-47.

9. Fornerod M, Ohno M, Yoshida M, Mattaj IW. CRMI is an export receptor for leucine-rich nuclear export signals. Cell 1997 ; 90: 1051-60.

10. Fukuda M, Asano S, Nakamura T, et al. CRMI is responsible for intracellular transport mediated by the nuclear export signal. Nature 1997; 390: 308-11.

11. Ossareh-Nazari B, Bachelerie F, Dargemont C. Evidence for a role of CRMI in signal-mediated nuclear protein export. Science 1997; 278: 141-4.

12. Stade K, Ford CS, Guthrie C, Weis K. Exportin 1 (CRMlp) is an essential nuclear export factor. Cell 1997; 90: 1041-50.

13. Kutay U, Bischoff FR, Kostka S, Kraft R, Gorlich D. Export of importin a from the nucleus is mediated by a specific nuclear transport factor. Cell 1997; 90: 1061-71.

14. Lipowsky G, Bischoff FR, Schwarzmaier $P$, et al. Exportin 4 : a mediator of a novel nuclear export pathway in higher eukaryotes. EMBO J 2000 ; 19: 4362-71.

15. Brownawell AM, Macara IG. Exportin 5, a novel karyopherin, mediates nuclear export of doublestranded RNA binding proteins. J Cell Biol 2002; 156: 53-64. 
16. Yoshida K, Blobel G. The karyopherin Kap142p/Msn5p mediates nuclear import and nuclear export of different cargo proteins. J Cell Biol 2001; 152: 729-40.

17. Mingot JM, Kostka S, Kraft R, Hartmann $\varepsilon$, Gorlich D. Importin 13: a novel mediator of nuclear import and export. EMBO J 2001; 20 : 3685-94.

18. Black BE, Holaska JM, Rastinejad F, Paschal BM. DNA binding domains in diverse nuclear receptors function as nuclear export signals. Curr Biol 2001; 11: 1749-58.

19. Azuma Y, Dasso M. The role of Ran in nuclear function. Curr Opin Cell Biol 2000; 12 : 302-7.

20. Lindsay ME, Holaska JM, Welch K, Paschal BM, Macara IG. Ran-binding protein 3 is a cofactor for CRMlmediated nuclear protein export. J Cell Biol 2001; 153: 1391-402.

21. Rexach M, Blobel G. Protein import into nuclei: association and dissociation reactions involving transport substrate, transport factors, and nucleoporins. Cell 1995; 83: 683-92.

22. Allen NP, Huang $L$, Burlingame A, Rexach MF. Proteomic analysis of nucleoporin interacting proteins. J Biol Chem 2001; 153: 29268-74.

23. Rout MP, Aitchison JD, Suprapto A, Hjertaas K, Zhao $y$, Chait BT.

The yeast nuclear pore complex: composition, architecture, and transport mechanism. J Cell Biol 2000; 148: 635-51.

24. Ribbeck K, Gorlich D. Kinetic analysis of translocation through nuclear pore complexes. EMBO J 2001; 20 : 1320-30.

25. Kehlenbach RH, Dickmanns A, Kehlenbach A, Guan T, Gerace L. A role for RanBPI in the release of CRMI from the nuclear pore complex in a terminal step of nuclear export. J Cell Biol 1999; 145: 645-57.
26. Black BE, Holaska JM, Levesque $L$, et al. NXTl is necessary for the terminal step of CRMI-mediated nuclear export. J Cell Biol 2001; 152: 141-55.

27. Wu J, Matunis MJ, Kraemer D, Blobel G, Coutavas $\varepsilon$. Nup358, a cytoplasmically exposed nucleoporin with peptide repeats, Ran-GTP binding sites, zinc fingers, a cyclophilin A homologous domain, and a leucine-rich region. J Biol Chem 1995; 270: 14209-13.

28. Yokoyama N, Hayashi N, Seki $T$, et al. A giant nucleopore protein that binds Ran/TC4. Nature 1995; 376: 184-8.

29. Mahajan R, Delphin C, Guan T, Gerace L, Melchior F. A small ubiquitin-related polypeptide involved in targeting RanGAPl to nuclear pore complex protein RanBP2. Cell 1997; 88: 97-107.

30. Karin M, Ben-Neriah y. Phosphorylation meets ubiquitination: the control of NF-KB activity. Annu Rev Immunol 2000; 18: 621-63.

31. Arenzana-Seisdedos F, Turpin P, Rodriguez M, et al. Nuclear localization of $\mid \kappa B \alpha$ promotes active transport of NF- $\kappa B$ from the nucleus to the cytoplasm. J Cell Sci 1997; 110: 369-78.

32. Johnson C, Van Antwerp D, Hope TJ. An N-terminal nuclear export signal is required for the nucleocytoplasmic shuttling of $\mid \kappa B \alpha$. EMBO J 1999; 18: 6682-93.

33. Tam WF, Lee LH, Davis L, Sen R. Cytoplasmic sequestration of rel proteins by $1 \kappa B \alpha$ requires CRM-dependent nuclear export. Mol Cell Biol 2000; 20: 2269-84.
34. Rodriguez MS, Thompson J, Hay RT, Dargemont C. Nuclear retention of $\mathrm{I} \kappa \mathrm{B} \alpha$ protects it from signal induced degradation and inhibits NF- $\mathrm{KB}$ transcriptional activation. J Biol Chem 1999; 274 : 9108-15.

35. Yang J, Kornbluth S. All aboard the cyclin train: subcellular trafficking of cyclins and their $\mathrm{Cdk}$ partners. Trends Cell Biol 1999; 9: 207-10.

36. Hagting $A$, Karlsson C, Clute $P$, Jackman M, Pines J. MPF localization is controlled by nuclear export. EMBO J 1998; 17: 4127-38.

37. Toyoshima F, Moriguchi T, Wada A, Fukuda M, Nishida $\varepsilon$. Nuclear export of cyclin $\mathrm{Bl}$ and its possible role in the DNA damage-induced G2 checkpoint. EMBO J 1998; 17: 2728-35.

38. Yang J, Bardes ES, Moore JD, Brennan J, Powers MA, Kornbluth $S$. Control of cyclin Bl localization through regulated binding of the nuclear export factor CRM1. Genes Dev 1998; 12 : 2131-43.

39. Toyoshima-Morimoto $F$, Taniguchi $\varepsilon$, Shinya $N$, Iwamatsu A, Nishida $\varepsilon$. Polo-like kinase 1 phosphorylates cyclin Bl and targets it to the nucleus during prophase. Nature 2001; 410: 215-20.

40. Lopez-Girona A, Furnari B, Mondesert 0 , Russell $P$. Nuclear localization of Cdc25 is regulated by DNA damage and a 14-3-3 protein. Nature 1999; 397 : 172-5.
41. Graves PR, Lovly CM, Uy GL, Piwnica-Worms $\mathrm{H}$. Localization of human Cdc25C is regulated both by nuclear export and 14-3-3 protein binding. Oncogene 2001; 20: 1839-51.

42. Pruyne D, Bretscher $A$. Polarization of cell growth in yeast. I. Establishment and maintenance of polarity states. J Cell Sci 2000; 113: 365-75.

43. Gulli MP, Peter M. Temporal and spatial regulation of Rho-type guaninenucleotide exchange factors: the yeast perspective. Genes Dev 2001; 15: 365-79.

44. Nern A, Arkowitz RA. Nucleocytoplasmic shuttling of the $\mathrm{Cdc} 42 \mathrm{p}$ exchange factor $\mathrm{Cdc} 24 \mathrm{p}$. J Cell Biol 2000; 148: 1115-22.

45. Henchoz S, Chi Y, Catarin B, Herskowitz I, Deshaies RJ, Peter M. Phosphorylationand ubiquitin-dependent degradation of the cyclindependent kinase inhibitor Farlp in budding yeast. Genes Dev 1997; 11 : 3046-60.

46. Blondel M, Galan JM, Chi Y, et al. Nuclear-specific degradation of Farl is controlled by the localization of the F-box protein Cdc4. EMBO J 2000; 19: 6085-97.

47. Blondel M, Alepuz PM, Huang LS, Shaham S, Ammerer G, Peter $M$. Nuclear export of Farlp in response to pheromones requires the export receptor Msn5p/Ste2lp. Genes Dev 1999; 13: 2284-300. 